\title{
Optimization of odd chain fatty acid production by Yarrowia lipolytica
}

\author{
Young-Kyoung Park', Thierry Dulermo ${ }^{1,2}$, Rodrigo Ledesma-Amaro ${ }^{1,3^{*}}$ and Jean-Marc Nicaud ${ }^{1 *}$ (D)
}

\begin{abstract}
Background: Odd chain fatty acids (odd FAs) have a wide range of applications in therapeutic and nutritional industries, as well as in chemical industries including biofuel. Yarrowia lipolytica is an oleaginous yeast considered a preferred microorganism for the production of lipid-derived biofuels and chemicals. However, it naturally produces negligible amounts of odd chain fatty acids.

Results: The possibility of producing odd FAs using Y. lipolytica was investigated. Y. lipolytica wild-type strain was shown able to grow on weak acids; acetate, lactate, and propionate. Maximal growth rate on propionate reached $0.24 \pm 0.01 \mathrm{~h}^{-1}$ at $2 \mathrm{~g} / \mathrm{L}$, and growth inhibition occurred at concentration above $10 \mathrm{~g} / \mathrm{L}$. Wild-type strain accumulated lipids ranging from 7.39 to $8.14 \%$ (w/w DCW) depending on the carbon source composition, and odd FAs represented only $0.01-0.12 \mathrm{~g} / \mathrm{L}$. We here proved that the deletion of the PHD1 gene improved odd FAs production, which reached a ratio of $46.82 \%$ to total lipids. When this modification was transferred to an obese strain, engineered for improving lipid accumulation, further increase odd FAs production reaching a total of $0.57 \mathrm{~g} / \mathrm{L}$ was shown. Finally, a fed-batch co-feeding strategy was optimized for further increase odd FAs production, which generated $0.75 \mathrm{~g} / \mathrm{L}$, the best production described so far in Y. lipolytica.

Conclusions: A Y. lipolytica strain able to accumulate high level of odd chain fatty acids, mainly heptadecenoic acid, has been successfully developed. In addition, a fed-batch co-feeding strategy was optimized to further improve lipid accumulation and odd chain fatty acid content. These lipids enriched in odd chain fatty acid can (1) improve the properties of the biodiesel generated from Y. lipolytica lipids and (2) be used as renewable source of odd chain fatty acid for industrial applications. This work paves the way for further improvements in odd chain fatty acids and fatty acid-derived compound production.
\end{abstract}

Keywords: Yarrowia lipolytica, Oleaginous yeast, Biolipid, Propionate, Odd chain fatty acids, Pentadecanoic acid, Heptadecanoic acid, Heptadecenoic acid, Metabolic engineering

\section{Background}

With the increasing environmental and energy concern, microbial oils (lipids and fatty acid-derived products) are regarded as promising alternatives to fossil fuels that can be used for the production of biofuels and oleo-chemicals. Microbial oils present multiple advantages over plant oils or animal fats, because they are not competitive

\footnotetext{
${ }^{*}$ Correspondence: r.ledesma-amaro@imperial.ac.uk; jean-marc.nicaud@inra.fr

1 UMR1319, Team BIMLip: Biologie Intégrative du Métabolisme Lipidique, Institut Micalis, INRA-AgroParisTech, Université Paris-Saclay, Domaine de Vilvert, 78352 Jouy-en-Josas, France

Full list of author information is available at the end of the article
}

with food, are less susceptible to seasonal availability, and they can be engineered to tune their composition and, therefore, properties [1]. For these reasons, many attempts have taken place to enhance lipid production from microorganisms with diverse metabolic engineering approaches. Despite the enhancement of microbial oils production, costs are not low enough to make the process economically feasible. One way to reduce production costs is to use low-cost carbon substrates [2]. Another is to produce value-added lipids or chemicals not readily obtainable via traditional petrochemical processes [3]. An example of this would be the production of odd chain fatty acids. 
Microbial lipids contain mostly fatty acids from 12 to 22 carbon atoms, with the prevalence of the even-numbered of 16-20 [4]. The availability of fatty acids with odd carbon number is scarce, although they are more valuable for commercialization because of their various applications [3]. For example, cis-9-heptadecenoic acid is known to have an anti-inflammatory effect and actives on psoriasis, allergies, and autoimmune diseases [5]. Pentadecanoic acid and heptadecanoic acid can be used as biomarkers for dietary food intake assessment, coronary heart disease (CHD) risk, and type II diabetes mellitus risk [6-9]. The chemical properties and potential biological activities of odd chain fatty acids are now being more extensively studied [4], so novel nutritional and pharmaceutical application could be discovered. The biodiesel properties directly depend on the fatty acid composition of biodiesel fuel [10]. Importantly, and although most effect is caused by the saturated/unsaturated fatty acid ratio, odd chain fatty acids also have a positive impact in the quality of biofuels enhancing transesterification reactions or storage conditions. In addition to fuels, the odd chain fatty acids and derivatives are precursors for manufacturing agricultural chemicals like biocides, flavor and fragrance intermediates, hydraulic fluids, plasticizers, coatings, and industrial chemicals [11-14].

Despite the wide range of application, studies aimed to produce odd chain fatty acids in microorganisms are limited because most of microbial cells normally produce even chain fatty acids. It is reported that exogenous propionate $(\mathrm{C} 3)$ can be used as a primer for synthesis of odd chain fatty acids: Propionate can be converted to propionyl-CoA by propionyl-CoA synthase, and propionyl-CoA is condensed with malonyl-CoA in the first step of odd chain fatty acid synthesis [15]. A metabolic engineering strategy with propionate supplementation achieved a production of $0.276 \mathrm{~g} / \mathrm{L}$ odd chain free fatty acids in E. coli [16]. In addition, further engineering of $E$. coli showed an increased percentage of odd chain free fatty acids in total free fatty acids by 6.25 -fold with propionate supplementation [17]. Odd chain fatty acids have also been produced, with propionate supplementation, in both oleaginous yeasts (Candida sp., Rhodotorula glutinis, Trichosporon cutaneum, Y. lipolytica, Cryptococcus curvatus) and non-oleaginous yeast (Kluyveromyces polysporus, Saccharomyces cerevisiae, Torulaspora delbrueckii) $[18,19]$. In the best performing yeast, Y. lipolytica, odd chain fatty acid did not exceed about 30\% of total lipid with a maximum total lipid production of $0.31 \mathrm{~g} / \mathrm{L}$, and the highest lipid content of $8.9 \% \mathrm{~g} / \mathrm{g}$ of cell dry weight $(\mathrm{CDW})$ [18]. The studies on odd chain fatty acids production in yeast have been done, so far, by optimizing fermentation conditions [19] or evaluating capacity of producing lipids among several strains [18]. More research on propionate utilization and metabolic engineering approaches for enhancing odd chain fatty acids production are, therefore, needed.

Propionic acid is an abundant volatile fatty acid (VFA) which can be obtained from agro-industrial lignocellulosic wastes, sludge and several biodegradable organic wastes [20]. Recently, VFAs is gaining interests as a substrate for lipid production by oleaginous microorganisms since it can be produced from wastes with low-costs [18, 21, 22]. Additionally, VFAs including propionate could lead to higher theoretical conversion efficiencies to lipids compared to other sugar-based carbon sources such as glucose and glycerol due to their shorter metabolic pathways [19, 23]. Studies on tolerance and utilization of propionic acid by oleaginous yeast for lipid production are still limited in the literature, it is sure that further research are necessary to use VFAs, either propionate alone or mixture of VFAs, as more feasible carbon sources.

Yarrowia lipolytica is a widely recognized oleaginous yeast known for its superior characteristics in the production of lipids and fatty acid-derived compounds, as well as other biotechnological products such as organic acids, nutraceuticals, emulsifiers, and surfactants [2, 24]. In addition, $Y$. lipolytica can grow in a broad range of substrates and it has been recently engineered for expanding the substrates range of this yeast including renewable biomass. Several strategies by overexpressing genes or deleting competitive pathway have also been used for improving even lipid accumulation in Y. lipolytica [25-27].

In this work, we investigated the ability of $Y$. lipolytica to produce odd chain fatty acids from propionate either as sole carbon and energy source or in combination with glucose. To increase the propionyl-CoA pool for the synthesis of odd chain fatty acids, we disrupted PHD1 encoding 2-methylcitrate dehydratase in the methyl citrate cycle. We also engineered the strain to accumulate more fatty acid by enhancing the synthesis capacity and blocking the degradation of lipids. Additionally, a fedbatch co-feeding strategy with glucose and propionate further increased total odd chain fatty acids. This work paves the way to use Y. lipolytica as a platform microorganism for producing valuable biochemicals with oddnumbered carbon chain.

\section{Results and discussion}

\section{Yarrowia lipolytica can grow on propionate as sole carbon source}

It is known that $Y$. lipolytica can be grown using diverse carbon sources from hydrophobic substrates such as $n$-alkanes, fatty acids, and oils to hydrophilic ones such as sugars or organic acids [21]. It was also reported that 
VFAs could be used as substrates for lipid production $[18,21,28]$. However, high concentration of VFA and weak acids inhibit cell growth which differs depending on strains $[18,21,28,29]$. The $Y$. lipolytica strains used in this study were derived from the wild-type $Y$. lipolytica W29 strain (ATCC20460). The auxotrophic derivative Po1d (Leu- Ura-, Table 1, Additional file 1: Figure S1) was previously described by Barth and Gaillardin [30]. The Po1d prototroph derivative JMY2900 (Table 1) was used as wild-type reference strain for the comparison with engineered strains derived from Pold [31]. Growth performance of our reference strain on weak acids and inhibitory effect of propionate were analyzed in microplate (Fig. 1). Y. lipolytica was able to grow on weak acids at similar growth rate, about $0.16 \mathrm{~h}^{-1}$, lower than in glucose $\left(0.25 \mathrm{~h}^{-1}\right)$ (Fig. 1a, Additional file 1: Table S1). As previously shown, Y. lipolytica can utilize propionate as a sole carbon source-a substrate that promotes odd chain

Table 1 E. coli and Y. lipolytica strains used in this study

\begin{tabular}{|c|c|c|}
\hline Name & Relevant genotype/plasmid description & Source of references \\
\hline \multicolumn{3}{|l|}{ E. coli strains } \\
\hline $\mathrm{DH} 5 \mathrm{a}$ & $\begin{array}{l}\text { Ф80lacZ } \Delta \mathrm{m} 15 \Delta\left(\text { lacZYA-argF) U169 recA1 endA1 hsdR17 }\left(r_{\mathrm{k}}^{-}, \mathrm{m}_{\mathrm{k}}^{+}\right) \text {phoA supE44 thi-1 }\right. \\
\text { gyrA96 relA1 } \lambda^{-}\end{array}$ & Promega \\
\hline JME740 & DH5a pKS-PUT phd1 & {$[32]$} \\
\hline JME547 & DH5a pUB4-CRE1 & [53] \\
\hline JME1000 & DH5a pKS-PLT tgl4 & {$[37]$} \\
\hline JME1077 & DH5a pGEMT easy-PUT mfe 1 & {$[36]$} \\
\hline JME1128 & DH5a JMP62-pTEF-GPD1-URA3ex & {$[36]$} \\
\hline JME1822 & DH5a JMP62-pTEF-DGA2-LEU2ex & [38] \\
\hline \multicolumn{3}{|l|}{ Y. lipolytica strains } \\
\hline Po1d (JMY195) & MATa ura3-302 leu2-270 xpr2-322 & {$[30]$} \\
\hline JMY2900 & Po1d Ura+ Leu+ & [31] \\
\hline JMY1203 & Pold phd1::URA3ex & [32] \\
\hline JMY3279 & Po1d $\Delta p h d 1$ & This study \\
\hline JMY3348 & Po1d $\triangle p h d 1$ mfe1::URA3ex & This study \\
\hline JMY3350 & Po1d phd1::URA3ex + LEU2 & This study \\
\hline JMY3396 & Pold $\triangle$ phd1 mfel::URA3ex tgl4::LEU2ex & This study \\
\hline JMY3433 & Pold $\Delta p h d 1 \Delta m f e 1 \Delta t g / 4$ & This study \\
\hline JMY3576 & Po1d $\Delta p h d 1 \triangle m f e 1 \Delta t g / 4+p T E F-D G A 2-L E U 2 e x$ & This study \\
\hline JMY3776 & Po1d $\Delta p h d 1 \Delta m f e 1 \Delta t g / 4+p T E F-D G A 2-L E U 2 e x+p T E F-G P D 1-U R A 3 e x$ & This study \\
\hline
\end{tabular}
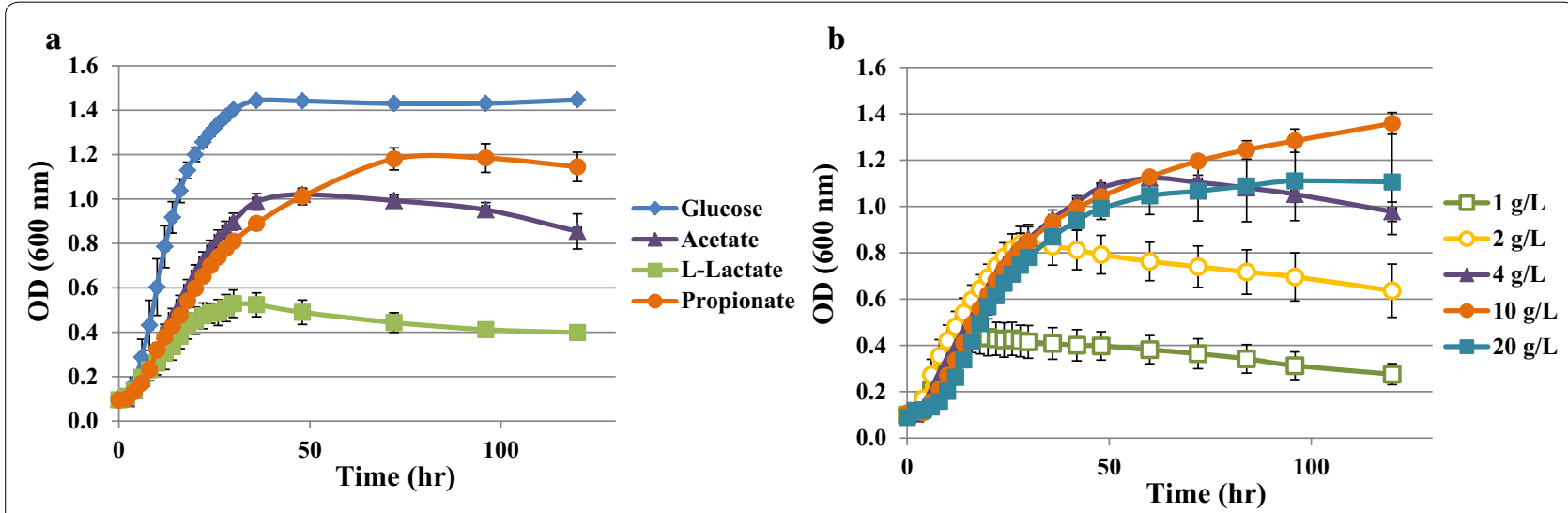

Fig. 1 Growth of wild-type W29 derivative strain JMY2900 on weak acids and on different propionate concentration. a Cell growth in minimal media containing $5 \mathrm{~g} / \mathrm{L}$ of glucose, acetate, L-lactate and propionate as a sole carbon source. b Cell growth in propionate medium from 1 to $20 \mathrm{~g} / \mathrm{L}$. Growth curves are representative of three independent tests 
fatty acid production. Our reference $Y$. lipolytica strain JMY2900 was able to grow on propionate although the growth rate and the final OD were lower than in glucose (Fig. 1a). This growth inhibition was also shown in other organic acids in the following order: L-lactate > propionate $>$ acetate. In comparison to acetate, growth on propionate showed lower growth rate $\left(0.16 \mathrm{~h}^{-1}\right)$ but higher final OD at same concentration $(5 \mathrm{~g} / \mathrm{L})$. Although acetate has been regarded as preferable carbon source among VFAs because of their relatively lower growth inhibitory effect in previous studies [21,28], our results showed that propionate can be also a potential carbon source for biomass and lipid production in our Y. lipolytica strain.

In a previous study, it is reported that propionate has an inhibitory effect on the cell growth at concentrations above $5 \mathrm{~g} / \mathrm{L}$ [21]. To explore if our strain could grow on higher concentration than $5 \mathrm{~g} / \mathrm{L}$, JMY2900 was cultivated with different concentrations of propionate (Fig. 1b, Additional file 1: Figure S2). Our strain was able to grow up to $100 \mathrm{~g} / \mathrm{L}$ of propionate as a sole carbon source, the highest growth rate was observed at $2 \mathrm{~g} / \mathrm{L}$ of propionate (Additional file 1: Table S2). There was no big difference in initial OD trends between 4 and $10 \mathrm{~g} / \mathrm{L}$ of propionate, but JMY2900 on $10 \mathrm{~g} / \mathrm{L}$ of propionate was able to grow at higher cell density. The inhibitory effect of propionate to the cell growth was observed on concentration higher than $10 \mathrm{~g} / \mathrm{L}$ (Additional file 1: Table S2). The growth test at higher concentration of propionate $(100 \mathrm{~g} / \mathrm{L})$ showed a long lag phase of more than $48 \mathrm{~h}$ (Additional file 1: Figure S2), this shows propionate can be used as a carbon source in our strain. However, several Y. lipolytica strains behave very differently. For example, our strain appeared to be less sensitive to propionate than Y. lipolytica strain CICC31596 which shows an inhibitory effect of propionate on growth rate and lag phase already at $5 \mathrm{~g} / \mathrm{L}$ [28], while $Y$. lipolytica strain ISA 1834 showed higher growth rate, $0.29 \mathrm{~h}^{-1}$, on propionate [29]. This demonstrates important differences in propionate sensitivity depending on either strains, culture conditions or media composition.

\section{Accumulation of odd chain fatty acids in propionate medium}

To see whether propionate is a suitable carbon source for odd lipid production, flask cultures of JMY2900 with several compositions of carbon sources (YNBD1 glucose 1\%, YNBD1P1 glucose $1 \%$ and propionate $1 \%$, YNBP1 propionate $1 \%$, YNBP2 propionate $2 \%$ ) were carried out. It was revealed that lipid content obtained on YNBP1 (7.48\%) was comparable to that on YNBD1 (7.86\%) with significant difference of lipids composition (Table 2, Fig. 2). In YNBD1 media, oleic acid (C18:1) and linoleic acid (C18:2) were the major products with a percentage of

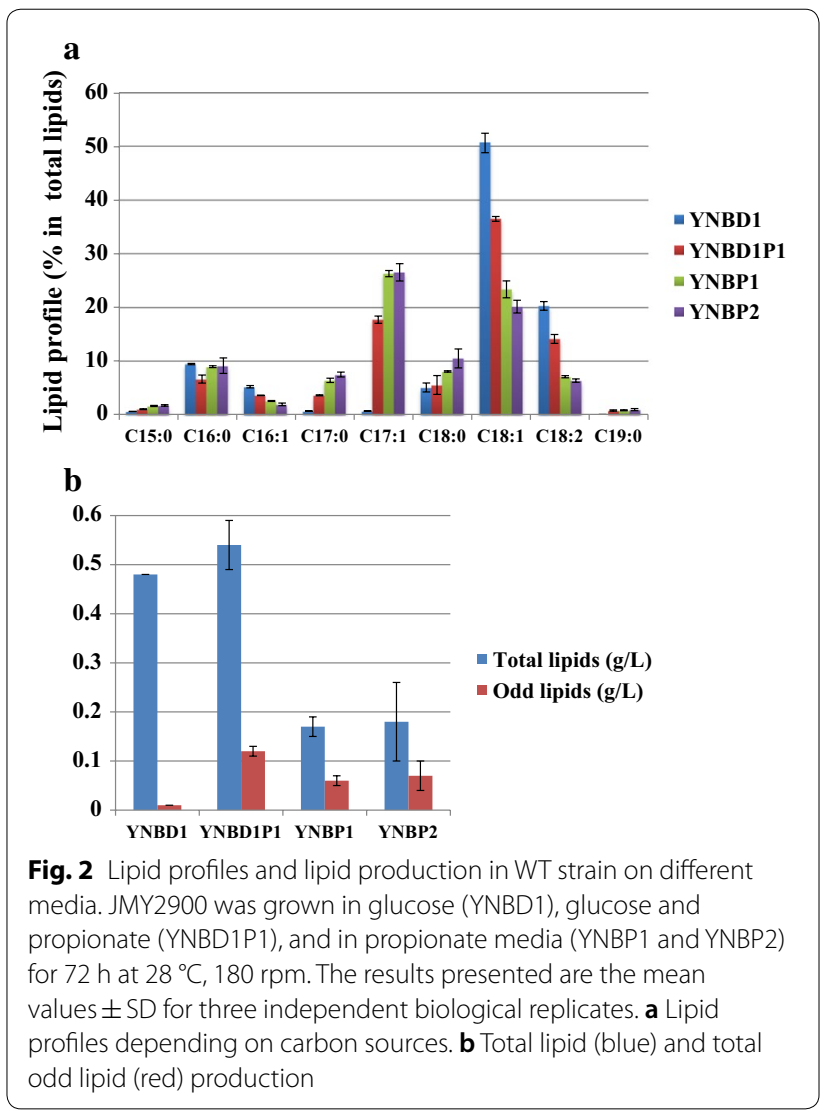

Table 2 Biomass and lipid production by wild-type strain JMY2900 in minimal medium

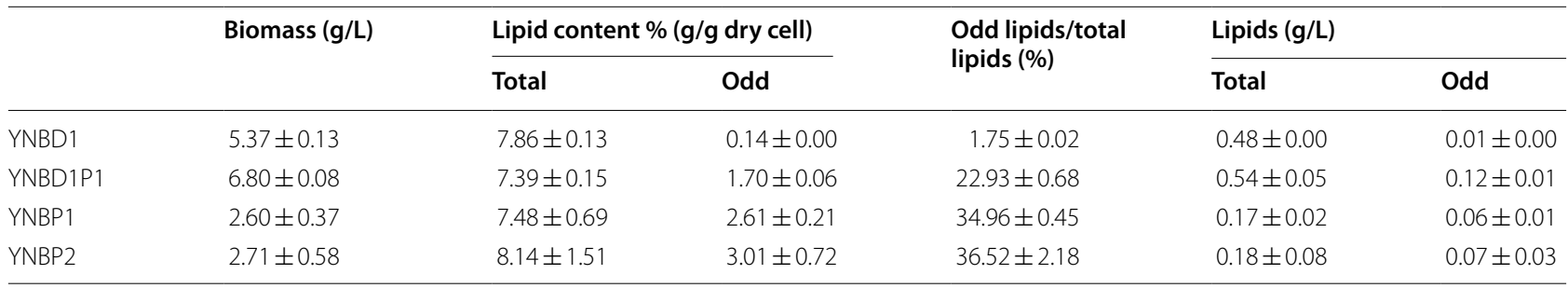

Strain was grown for $72 \mathrm{~h}$ at $28^{\circ} \mathrm{C}$ and $180 \mathrm{rpm}$

The mean value of three independent experiments is shown and the standard deviation is indicated 
50.72 and $20.26 \%$, respectively. Only $1.75 \%$ of odd chain fatty acids in total fatty acids were produced in this condition. However, in case of medium without glucose like YNBP1 and YNBP2, the ratio of odd chain fatty acid to total lipids increased to around 35\%. In these conditions, the portion of oleic acid and linoleic acid in total lipids decreased in contrast to the increase of heptadecenoic acid (C17:1). In addition to heptadecenoic acid (C17:1), other odd chain fatty acids such as pentadecanoic acid (C15:0), heptadecanoic acid (C17:0), and nonadecanoic acid (C19:0) were also produced from all propionate-containing medium (Table 2, Fig. 2a). These results indicate that propionate can be used as a primer for the synthesis of odd chain fatty acids in Y. lipolytica as reported in other studies $[18,19]$. Although the total lipid contents from YNBD1 and YNBP1 are similar, the biomass produced was significantly different $(5.37$ and $2.60 \mathrm{~g} / \mathrm{L}$, respectively). The difference in biomass production in YNBP1 and YNBP2 was already shown in Fig. 1b, and it might be due to a higher the inhibitory effect of higher concentration of propionate. In spite of lower ratio of odd chain fatty acids to total fatty acids in YNBD1P1 than that of YNBP1, $0.12 \mathrm{~g} / \mathrm{L}$ of odd chain fatty acid was produced which showed the highest amount in this culture (Table 2). JMY2900 accumulated slightly higher odd and total lipids in YNBP2 than YNBP1, but it did not show significantly better performance for odd chain fatty acids production. In addition, higher concentration of propionate showed inhibitory effect from the beginning of culture (data not shown). From these results, YNBD1P1 is the best condition for the odd and total lipids production, and YNBP1 is also a suitable condition for high ratio of odd chain fatty acids to total fatty acids (Fig. 2b).

\section{Inactivation of the propionate catabolic pathway improved} odd chain fatty acid content and production

We previously reported the importance of the methylcitrate cycle on glycerol metabolism in Y. lipolytica [32]. PHD1, involved in the synthesis of 2-methylcitrate dehydratase is a mitochondrial protein, which catalyzes the conversion of 2-methyl citrate to 2-methyl-cis-aconitate in the methyl citrate cycle. It has been shown that, in $Y$. lipolytica, the deletion of PHD1 results in the accumulation of 2-methyl citrate, which could potentially halt the TCA cycle and inhibit the entry of citrate into mitochondria [32]. Additionally, deletion of the PHD1 gene coding for the 2-methyl-citrate dehydratase was shown to improved lipid accumulation. As described above, propionate can be converted to propionyl-CoA, which promotes the production of odd chain lipids. In $Y$. lipolytica, propionyl-CoA can be catabolized to form pyruvate and succinate through the methyl citrate cycle (Fig. 3). So the methyl citrate cycle can be regarded as competitive pathway for the synthesis of odd chain fatty acids. We hypothesize here that inhibition of 2-methyl citrate pathway by deleting PHD1 increases the propionyl-CoA pool that could be used for further synthesis of odd chain fatty acids. To prove this, culture of JMY3350 (WT $\Delta$ phd1) was performed in the same condition as above. As expected, JMY3350 was not able to grown in YNBP1 since that propionate cannot be used as a sole carbon source. This confirmed that propionate cannot be metabolized through methyl citrate cycle to form pyruvate in JMY3350. In glucose media (YNBD1), we observed an increased of the ratio of odd chain fatty acid to total fatty acid even without propionate by 1.35 times (Additional file 1: Table S3). Inactivation of PHD1 blocks the TCA cycle [32], which might cause growth defects and increase sensitivity of propionate to the cell. Therefore, we added lower amount of propionate $(4 \mathrm{~g} / \mathrm{L})$ with glucose $(10 \mathrm{~g} / \mathrm{L})$ after $16 \mathrm{~h}$ of the start of the culture with glucose (YNBD1). To compare the capability of the two strains, JMY2900 (WT) and JMY3350 (WT $\Delta p h d 1$ ), for odd chain fatty acid production, we used an equivalent amount of metabolizable carbon with a $\mathrm{C} / \mathrm{N}$ ratio 30, which is often found as the optimum condition for lipid production in Y. lipolytica [33-35]. Therefore, the glucose amount was adjusted in JMY3350 strain to compensate the lack of use of propionate as carbon source for biomass formation. The lipid content of JMY3350 increased by $17 \%$ comparing with the wildtype (8.01 and $6.85 \%)$. The ratio of odd chain fatty acids to total lipids was also higher (46.82\%) than the wild-type (28.32\%) (Table 3). JMY3350 produced $0.17 \mathrm{~g} / \mathrm{L}$ of odd chain fatty acids $21.4 \%$ higher than control strain despite of its lower biomass. The lower biomass formation has been previously reported for this mutant [32]. In addition, the inactivation of PHD1 modified the composition of lipids (Fig. 4). The percentage of heptadecanoic acid in total lipids increased 4 times. Heptadecenoic acid (C17:1) showed the highest portion (35.56\%) of total lipids in WT $\Delta p h d 1$ (JMY3350), while JMY2900 produced mostly oleic acid (44.75\%), likewise most of other Y. lipolytica strains. Stability of modified strains is a key parameter in a bioprocess. In this regard, $\Delta p h d 1$ strains is expected to be stable on time, since the gene was completely removed from genome and it is very unlikely that other enzymes evolve to consume propionate in a fermentation condition when glucose is the fed for growing.

\section{Engineering higher accumulation of odd chain FA}

Once we verified that strain carrying deletion of $P H D 1$ was able to produce more odd chain fatty acids, we wanted to engineer the strain to make it able to accumulate higher amount of total odd chain lipids. Therefore, we generated the strain named obese $\Delta p h d 1$ 


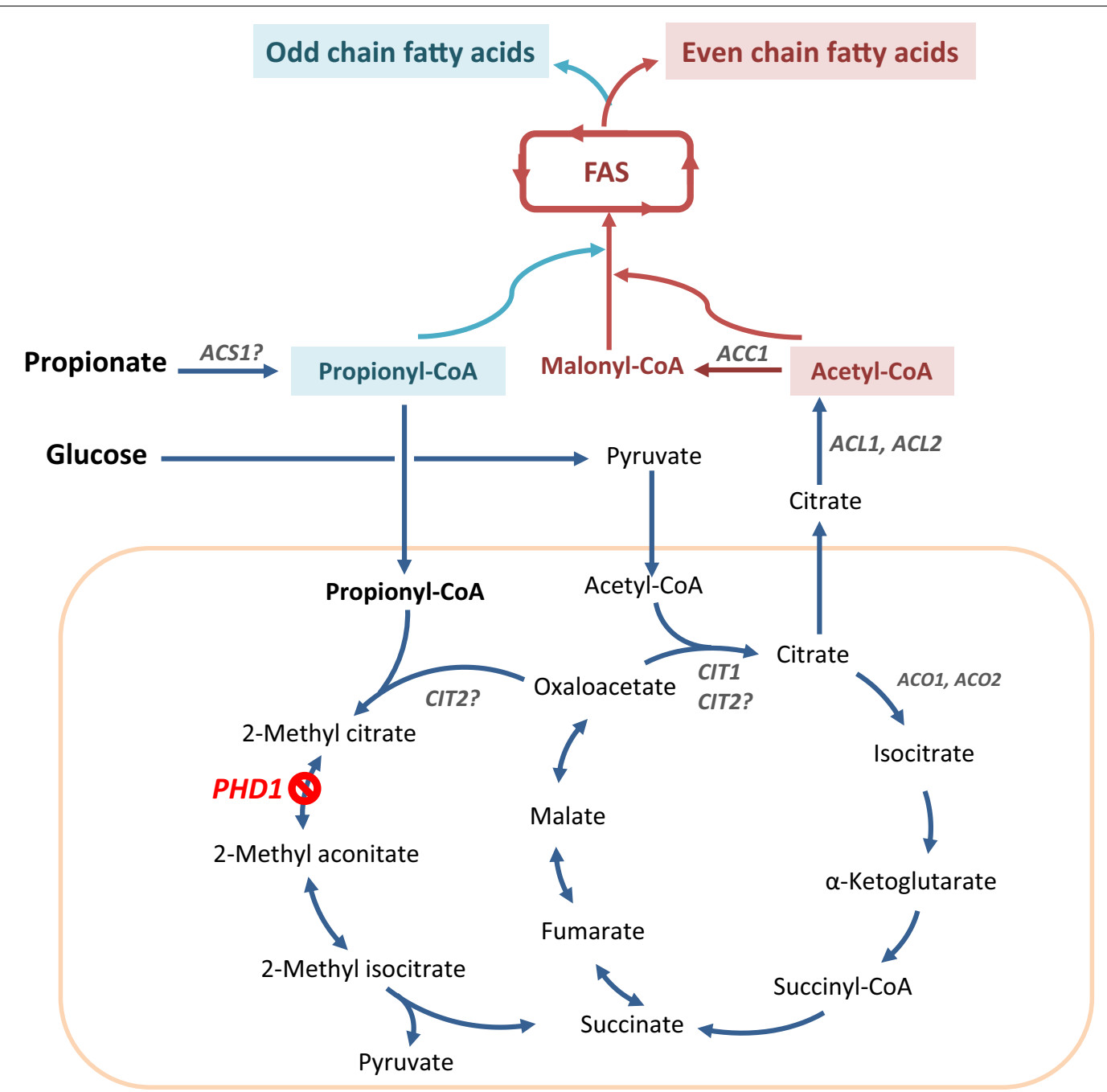

Fig. 3 Overview of the pathways involved in odd and even fatty acid production including the link with the TCA and methyl citrate cycles in Yarrowia lipolytica. Propionate is activated by the cytosolic acyl-CoA synthase (ACS1) to form the propionyl-CoA which is transported into mitochondria to enter the methyl citrate pathway. Propionate is condensed with oxaloacetate to form 2-methyl citrate by 2-methylcitrate synthase probably encoded by CIT2 (CIT2,YALIOE02684g). 2-Methyl citrate dehydratase removes an $\mathrm{H}_{2} \mathrm{O}$ to form 2-methyl aconitate by 2-methyl citrate dehydratase (PHD1, YALIOF02497), then is hydrated to form 2-methyl isocitrate probably by the aconitase (ACO1, YALI0D09361 g; ACO2, YALIOE14949g), which is cleaved by 2-methyl isocitrate lyase (YALIOF31999g) to give succinate and pyruvate. Glucose undergoes glycolysis and enters the mitochondria as a form of pyruvate to be used in the TCA cycle. Mitochondrial pyruvate is condensed with oxaloacetate by citrate synthase (CIT1, YALIOE00638g) to form citrate which can be exported to cytosol. The cytosolic citrate is transformed by the ATP-citrate lyase (subunit a, ACL1, YALIOE34793g and subunit b, ACL2, YALIOD24431g) into acetyl-CoA. Acetyl-CoA is then converted into malonyl-CoA by the acetyl-CoA carboxylase (ACC1, YALIOC1 1407g) as the first step of fatty acid synthesis. Acetyl-COA and malonyl-CoA are condensed by the fatty-acid synthase complex (FAS; subunit beta, FAS1, YALI0B15059g and subunit alpha, FAS2, YALIOB19382g) for the production of even fatty acids, while acetyl-CoA and propionyl-COA are condensed for the production of odd fatty acids

(JMY3776) by multiple modifications (Additional file 1 : Figure S1). First, to block $\beta$-oxidation, MFE1 encoding the multifunctional enzyme, involved in the second step of $\beta$-oxidation, was deleted [36]. To inhibit triacylglycerols (TAG) remobilization, TGL4 encoding a triglyceride lipase, was deleted [37]. In addition, to push and pull TAG biosynthesis, DGA2 encoding the major acylCoA: diacylglycerol acyltransferase [25, 38], and GPD1 encoding glycerol-3-phosphate dehydrogenase were overexpressed [36]. We then studied lipid production of engineered strain JMY3776 in the same conditions as before. As expected, odd chain lipid accumulation increased as well as total lipid accumulation, by 3.35 and 3.78 times, respectively (Table 3 ). The ratio of odd chain lipids to total lipids was slightly decreased in JMY3776, but still remained above $40 \%$. The amount of odd chain 
Table 3 Biomass and lipid production by WT, WT $\Delta p h d 1$ and obese $\Delta p h d 1$ strains in minimal glucose and propionate media

\begin{tabular}{|c|c|c|c|c|c|c|}
\hline & \multirow[t]{2}{*}{ Biomass (g/L) } & \multicolumn{2}{|c|}{ Lipid content \% (g/g dry cell) } & \multirow[t]{2}{*}{ Odd/total lipids (\%) } & \multicolumn{2}{|l|}{ Lipids (g/L) } \\
\hline & & Total & Odd & & Total & Odd \\
\hline JMY2900 (WT) & $7.18 \pm 0.25$ & $6.85 \pm 0.21$ & $1.94 \pm 0.03$ & $28.32 \pm 0.01$ & $0.49 \pm 0.01$ & $0.14 \pm 0.01$ \\
\hline JMY3350 (WT $\Delta p h d 1)$ & $4.50 \pm 0.50$ & $8.01 \pm 0.64$ & $3.75 \pm 0.04$ & $46.82 \pm 0.03$ & $0.36 \pm 0.01$ & $0.17 \pm 0.02$ \\
\hline JMY3776 (obese $\Delta p h d 1)$ & $5.53 \pm 0.32$ & $24.76 \pm 2.51$ & $10.37 \pm 0.49$ & $41.9 \pm 0.02$ & $1.36 \pm 0.06$ & $0.57 \pm 0.01$ \\
\hline
\end{tabular}

Strain JMY2900 (WT), JMY3350 (WT $\Delta p h d 1$ ) and JMY3776 (obese $\Delta p h d 1$ ) were grown for $72 \mathrm{~h}$ at $28^{\circ} \mathrm{C}$ and $180 \mathrm{rpm}$

The mean value of three independent experiments is shown and the standard deviation is indicated

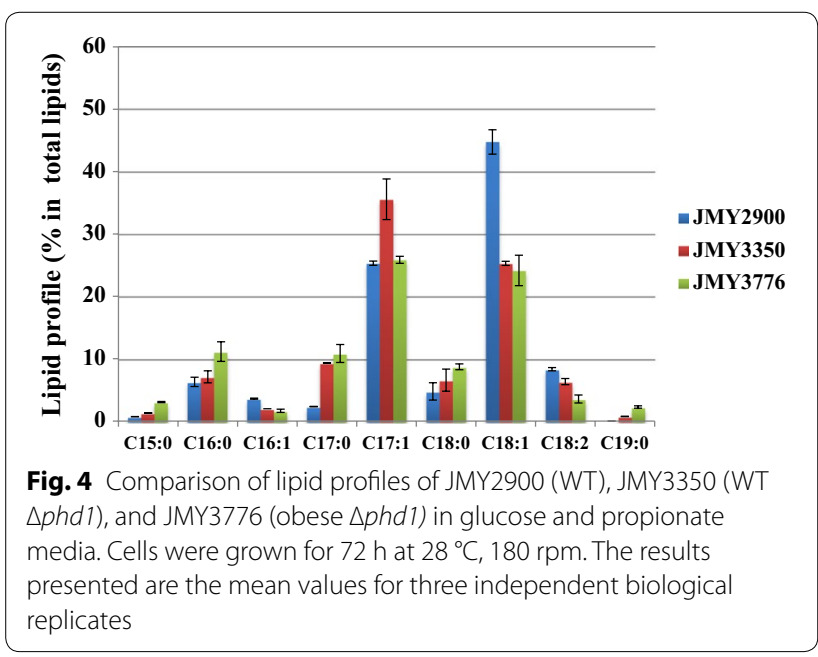

fatty acids was $0.57 \mathrm{~g} / \mathrm{L}$, the highest amount produced in Y. lipolytica, so far. The amount of all saturated fatty acids from C15:0 to C19:0 increased all together contrary to the unsaturated fatty acids moiety, which decreased (Fig. 4). This phenomenon was also shown in our previous study [39], the strains optimized for lipid accumulation (called obese strain) produced more C16:0 than wild-type and less unsaturated C16 and C18 commonly in different carbon sources (glucose, fructose, and sucrose).

\section{Increase of accumulation of odd FA by fed-batch co-feeding carbon sources}

As described above, propionate is a key carbon source for production of odd lipids meanwhile shows growth inhibitory effect in Y. lipolytica. Besides, the engineered strain is more sensitive to propionate allowing only small amount of propionate being used for odd chain lipid synthesis. Several fed-batch fermentation strategies have been used to improve yield and productivity by avoiding high level of inhibitory compounds in culture medium [40, 41]. To see whether fed-batch strategy could boost production of odd chain lipids while minimizing the inhibitory effect of propionate, we investigated fed-batch co-feeding of carbon sources during cultivation.

The obese $\Delta p h d 1$ strain was cultured in YNBD1 with addition of carbon sources (glucose $4 \mathrm{~g} / \mathrm{L}$ and propionate $0.5 \mathrm{~g} / \mathrm{L}$ ) at four times points (Fig. $5 \mathrm{a}$ ). As a result, the production of total lipid and odd chain lipid content, compare to batch culture, were increased by 50.35 and $12.64 \%$, respectively (Table 4 ). However, the percentage of odd chain lipids in total lipids is diminished by $60 \%$ as compared to batch condition likely due to the co-feeding with glucose. Nevertheless, the amount of total odd chain fatty acids from fed-batch co-feeding reached $0.75 \mathrm{~g} / \mathrm{L}, 31 \%$ higher than in batch, which represents the highest titer produced in Y. lipolytica so far (Additional file 1: Table S4). This represents a 395\% increase of odd chain fatty acid production between wild-type JMY2900 and the obese $\Delta p h d 1$ deleted strain in the fed-batch condition (Fig. 5b, c). Fed-batch fermentation has been beneficial for the production of other compounds by $Y$. lipolytica, such as the production of lipids described for the obese strain JMY3501 on synthetic media [42] or the obese strain producing carotenoides with the concomitant production of $42.6 \mathrm{~g} / \mathrm{L}$ of lipids on rich media [43]. The fermentation conditions can be further optimized by testing various feeding rate of glucose and propionate, which will allow to improve final biomass, higher lipid content and odd chain fatty acid content. Also, in future experiments it would be interesting to test other $\mathrm{C} / \mathrm{N}$ ratios, such as $\mathrm{C} / \mathrm{N}=60$ or $\mathrm{C} / \mathrm{N}=100$ which are found better for certain strains and conditions $[25,39,44,45]$.

Additionally, one could envisage the production of different types of odd chain fatty acids and their derivatives such as shorter odd chain fatty acid by engineering the fatty acid synthase (FAS) as recently demonstrated in Yarrowia lipolytica [46], hydroxylated odd chain fatty acid by introducing $\Delta 12$-hydroxylase (FAH12) from Claviceps purpurea [47] and odd chain dicarboxylic acid by overexpression of the omega 
a
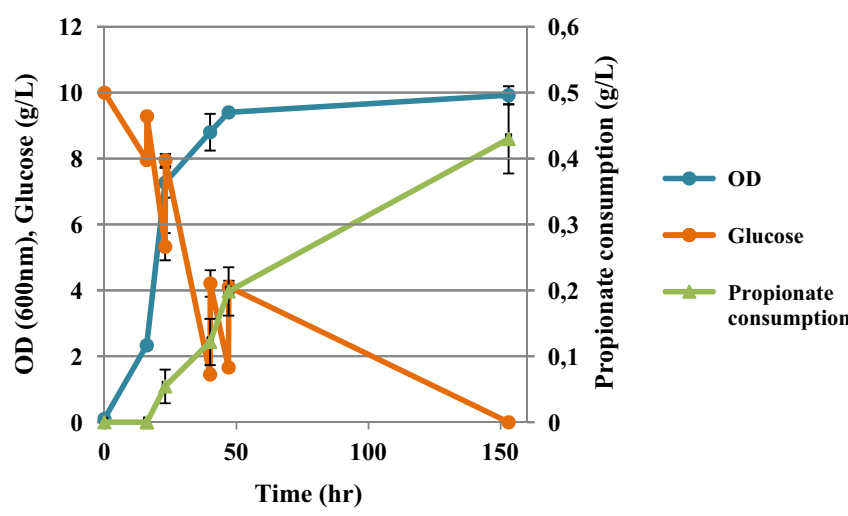

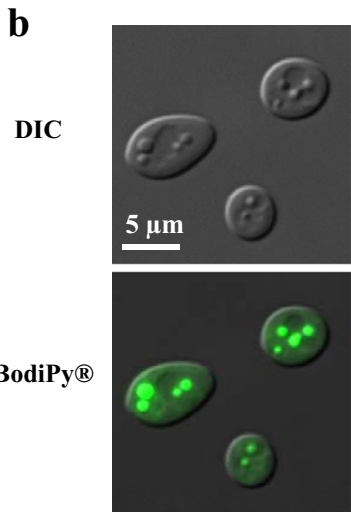

JMY2900
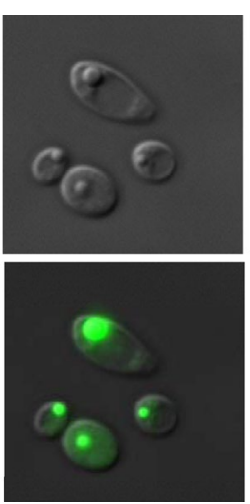

JMY3350

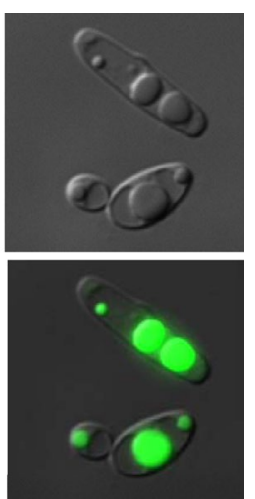

JMY3776 c

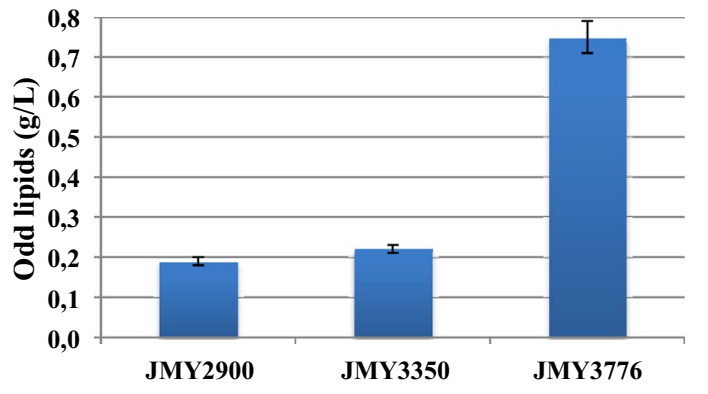

Fig. 5 Improvement of odd chain fatty acid production of JMY3776 by fed-batch co-feeding of propionate and glucose. a Increase of the accumulation of odd lipids by co-feeding of propionate and glucose. Cells were grown for $16 \mathrm{~h}$ in glucose media (YNBD1), then pulses of glucose + propionate ( 4 and $0.5 \mathrm{~g} / \mathrm{L}$, respectively) were added at $t=16,23,40,47 \mathrm{~h}$. The results presented are the mean values for three independent biological replicates. b Microscope image of cells (DIC) and lipid body visualization with Bodipy of JMY2900 (WT), JMY3350 (WT $\Delta p h$ 11) and JMY3776 (obese $\Delta$ phd1). c Increase of odd lipids accumulation by pathway engineering in fed-batch co-feeding cultures

Table 4 Biomass and lipid production by WT, WT $\Delta$ phd1 and obese $\Delta p h d 1$ strains by fed-batch co-feeding of glucose and propionate

\begin{tabular}{|c|c|c|c|c|c|c|}
\hline & \multirow[t]{2}{*}{ Biomass (g/L) } & \multicolumn{2}{|c|}{ Lipid content $\%$ (g/g dry cell) } & \multirow[t]{2}{*}{ Odd/total lipids (\%) } & \multicolumn{2}{|l|}{ Lipids (g/L) } \\
\hline & & Total & Odd & & Total & Odd \\
\hline JMY2900 (WT) & $8.20 \pm 0.05$ & $17.23 \pm 0.31$ & $2.30 \pm 0.02$ & $13.36 \pm 0.01$ & $1.41 \pm 0.02$ & $0.19 \pm 0.01$ \\
\hline JMY3350 (WT $\Delta p h d 1)$ & $4.83 \pm 0.13$ & $17.71 \pm 0.70$ & $4.58 \pm 0.05$ & $25.90 \pm 0.01$ & $0.85 \pm 0.01$ & $0.22 \pm 0.01$ \\
\hline JMY3776 (obese $\Delta p h d 1)$ & $5.93 \pm 0.13$ & $50.35 \pm 1.99$ & $12.64 \pm 0.45$ & $25.11 \pm 0.17$ & $2.99 \pm 0.18$ & $0.75 \pm 0.04$ \\
\hline
\end{tabular}

oxidation pathway [48]. However, further basic knowledge is required to fully understand propionic acid catabolism, its transport and toxicity to the cells.

\section{Conclusion}

In this study, it is shown that $Y$. lipolytica produce odd chain fatty acids (mainly heptadecenoic acid, 
heptadecanoic acid, and pentadecanoic acid) from propionate as a carbon source. By inactivating 2-methyl-citrate dehydratase in competing pathway utilizing propionyl$\mathrm{CoA}$, the amount of odd chain fatty acids is increased, the ratio of odd lipid to total lipids reached to $46.82 \%$. Obese $\Delta p h d 1$ strain engineered to accumulate higher amount of lipid produced 3.35 times higher odd chain lipids together with increased total lipid accumulation. In addition, a fed-batch co-feeding strategy further improved production of odd chain fatty acids with amount of $0.75 \mathrm{~g} / \mathrm{L}$ represents the highest titer produced in $Y$. lipolytica so far. Odd chain fatty acids are very important and versatile chemicals in both pharmaceutical and industrial fields. This work paves the way for further improvements in odd chain fatty acids and fatty acid-derived compound production.

\section{Methods}

\section{Strains and media}

Media and growth conditions for E. coli were as described by Sambrook et al. [49], and those for Y. lipolytica have been described by Barth and Gaillardin [30]. Rich medium (YPD) and minimal glucose medium (YNB) were prepared as described previously [50]. Minimal medium (YNB) contained $0.17 \%(\mathrm{w} / \mathrm{v})$ yeast nitrogen base (without amino acids and ammonium sulfate, YNBww, Difco), 0.5\% (w/v) $\mathrm{NH}_{4} \mathrm{Cl}, 50 \mathrm{mM}$ phosphate buffer ( $\mathrm{pH}$ 6.8). The following carbon sources were added: YNBD1 (1\% (w/v) glucose, YNBD1P1 (1\% (w/v) glucose, $1 \%(\mathrm{w} / \mathrm{v})$ propionate), YNBP1 $(1 \%(\mathrm{w} / \mathrm{v})$ propionate), YNBP2 (2\% (w/v) propionate). To complement auxotrophic processes, $0.1 \mathrm{~g} / \mathrm{L}$ of uracil or leucine (Difco) was added as necessary.

\section{Construction of strains}

The overexpression and disruption cassettes were prepared as described previously [36-38], and were used for transformation by the lithium acetate method [51]. Transformants were selected on YNBUra, YNBLeu, YNBHyg, YNB media depending on their genotype. Then genomic DNA from yeast transformants was prepared as described in Querol et al. [52]. Positive transformants were checked by PCR. The removal of the selection marker was carried out via the LoxP-Cre system as previously used in Y. lipolytica [53].

Restriction enzymes were obtained from New England Biolabs (Ipswich, MA, USA). PCR amplifications were performed in an Eppendorf 2720 thermal cycler with GoTaq DNA polymerases (Promega) and Q5 HighFidelity DNA Polymerase (New England Biolabs). PCR fragments were purified with a QIAgen Purification Kit (Qiagen, Hilden, Germany).

\section{Growth test}

Pre-cultures were inoculated into tubes containing $5 \mathrm{~mL}$ YPD medium, and cultured overnight $\left(28{ }^{\circ} \mathrm{C}, 180 \mathrm{rpm}\right)$. Pre-cultures were then centrifuged and washed with sterile distilled water, cell suspensions were standardized to an $\mathrm{OD}_{600}$ of 0.1. Stains were grown in $200 \mu \mathrm{L}$ of minimal YNB medium (see above) in the presence of carbon sources $(0.5 \%$ glucose, propionate, L-lactate, acetate as a carbon source) in 96-well plates, with constant shaking, at $28{ }^{\circ} \mathrm{C}$. Growth was monitored by measuring the optical density $\left(\mathrm{OD}_{600}\right)$ every $30 \mathrm{~min}$ for $120 \mathrm{~h}$ with a microtiter plate reader (Biotek Synergy MX, Biotek Instruments, Colmar, France). For each strain and set of conditions, we used three biological replicates. The growth rate was calculated in the exponential phase for each strain and condition.

\section{Culture conditions for lipid biosynthesis experiments}

For lipid biosynthesis in minimal media, cultures were prepared as follows: an initial pre-culture was established by inoculating $10 \mathrm{~mL}$ of YPD medium in $50 \mathrm{~mL}$ Erlenmeyer flasks. This was followed by an overnight shaking step at $28{ }^{\circ} \mathrm{C}$ and $180 \mathrm{rpm}$. The resulting cell suspension was washed with sterile distilled water and used to inoculate $50 \mathrm{~mL}$ of YNB medium containing $0.15 \%$ (w/v) $\mathrm{NH}_{4} \mathrm{Cl}$ and $50 \mathrm{mM}$ phosphate buffer ( $\mathrm{pH}$ 6.8) with various concentrations of carbon source in $250 \mathrm{~mL}$ Erlenmeyer flasks, at $28{ }^{\circ} \mathrm{C}$ and $180 \mathrm{rpm}$. For fed-batch co-feeding test, the strains were cultured in $20 \mathrm{~mL}$ of YNBD1 with addition $2 \mathrm{~mL}$ of mixture of carbon sources to a final concentration of glucose $4 \mathrm{~g} / \mathrm{L}$ and propionate $0.5 \mathrm{~g} / \mathrm{L}$ at four times points. The addition were performed at $T=16,23,40,48 \mathrm{~h}$ after the inoculation.

\section{Determination of glucose and propionate}

Glucose and propionate were identified and quantified by HPLC. Filtered aliquots of the culture medium were analyzed by UltiMate 3000 system (Thermo Fisher Scientific, UK) using an Aminex HPX-87H column (300 $\mathrm{mm} \times 7.8 \mathrm{~mm}$, Bio-RAD, USA) coupled to UV $(210 \mathrm{~nm})$ and RI detectors. The mobile phase used was $0.01 \mathrm{~N} \mathrm{H}_{2} \mathrm{SO}_{4}$ with a flow rate of $0.6 \mathrm{~mL} / \mathrm{min}$ and the column temperature was $T=35{ }^{\circ} \mathrm{C}$. Identification and quantification were achieved via comparisons to standards. For each data point, we used at least two biological replicates and calculated average and standard deviation values.

\section{Lipid determination}

Lipids were extracted from 10 to $20 \mathrm{mg}$ of freeze-dried cells, and converted into their fatty acid methyl esters (FAMEs) according to Browse et al. [54], and FAMEs 
were analyzed by gas chromatography (GC) analysis. GC analysis of FAMEs was carried out on a Varian 3900 instrument equipped with a flame ionization detector and a Varian FactorFour vf- $23 \mathrm{~ms}$ column, where the bleed specification at $260{ }^{\circ} \mathrm{C}$ is $3 \mathrm{pA}(30 \mathrm{~m}, 0.25 \mathrm{~mm}$, $0.25 \mu \mathrm{m})$. Fatty acids were identified by comparison to commercial FAME standards (FAME32, Supelco) and quantified by the internal standard method, involving the addition of $100 \mu \mathrm{g}$ of commercial dodecanoic acid (Sigma-Aldrich). Commercial odd chain fatty acids (9 Odd carbon fatty acids, OC9, Supelco) were converted to their FAMEs with a same method for yeast samples, and analyzed by GC to identify and compare odd chain fatty acids from yeast samples.

To determine DCW in flask experiments, $2 \mathrm{~mL}$ of the culture were washed and lysophilized in a pre-weighed tube. The differences in weight corresponded to the $\mathrm{mg}$ of cells found in $2 \mathrm{~mL}$ of culture. For each data point, we used at least two biological replicates and calculated average and standard deviation values.

\section{Microscopic analysis}

Images were obtained using a Zeiss Axio Imager M2 microscope (Zeiss, Le Pecq, France) with a $100 \times$ objective lens and Zeiss filter sets 45 and 46 for fluorescence microscopy. Axiovision 4.8 software (Zeiss, Le Pecq, France) was used for image acquisition. To make the lipid bodies (LBs) visible, BodiPy ${ }^{\circledR}$ Lipid Probe $(2.5 \mathrm{mg} / \mathrm{mL}$ in ethanol, Invitrogen) was added to the cell suspension $\left(\mathrm{OD}_{600}=5\right)$ and the samples were incubated for $10 \mathrm{~min}$ at room temperature.

\section{Additional file}

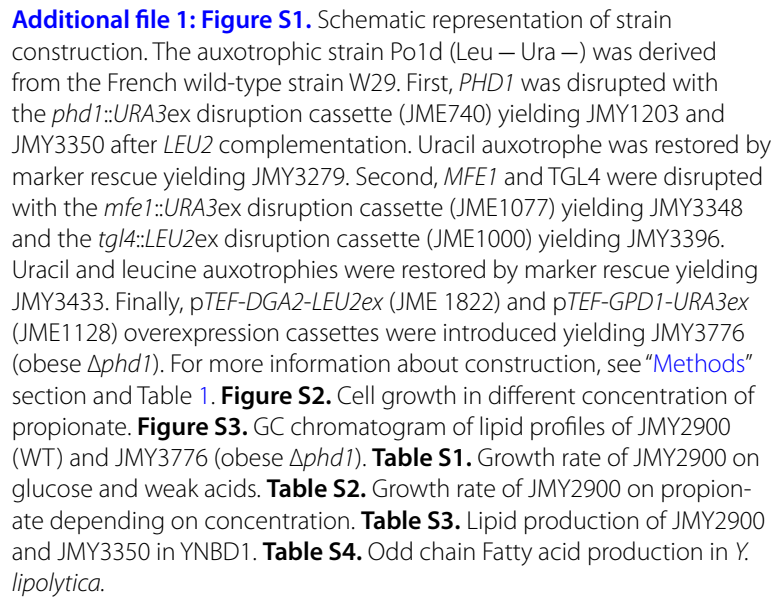

\section{Abbreviations}

DCW: dry cell weight; DIC: differential interference contrast; Odd FA: odd chain fatty acid; Even FA: even chain fatty acid; PCR: polymerase chain reaction.
Authors' contributions

YKP, RLA and JMN conceived the study and participated in its design. YKP, RLA designed the constructs, carried out all the experiments. TD designed and carried out the construction of the obese PHDI deleted strains. YKP drafted the manuscript. YKP, TD, RLA and JMN revised the manuscript. All authors read and approved the final manuscript.

\section{Author details}

${ }^{1}$ UMR1319, Team BIMLip: Biologie Intégrative du Métabolisme Lipidique, Institut Micalis, INRA-AgroParisTech, Université Paris-Saclay, Domaine de Vilvert, 78352 Jouy-en-Josas, France. ${ }^{2}$ Lesaffre International, Marcq-en-Baroeul, France. ${ }^{3}$ Department of Bioengineering, Imperial College London, London, UK.

\section{Acknowledgements}

The authors would like to thank Brigitte Thomasset (Université de Technologie de Compiegne) for the characterization of odd chain fatty acids by GC/MS.

\section{Competing interests}

The authors declare that they have no competing interests.

\section{Availability of supporting data}

All data generated or analyzed in the present study are included in this published article and a supporting material "Additional file 1".

\section{Consent for publication}

All authors consent for publication.

Ethical approval and consent to participate

Not applicable.

\section{Funding}

This work was partly funded by the Agence Nationale de la Recherche (Investissements d'Avenir program; reference ANR-11-BTBR-0003). Dr. LedesmaAmaro and Thierry Dulermo was a recipient of a postdoctoral fellowship from the Institut National de la Recherche Agronomique. Dr. Ledesma-Amaro received financial support from Imperial College London in the form of a IC Research Fellowship. Young-Kyoung Park was a recipient of an assistant engineered fellowship from the Institut National de la Recherche Agronomique and is the recipient of a PhD scholarship from the Kwanjeong Educational Foundation (KEF).

\section{Publisher's Note}

Springer Nature remains neutral with regard to jurisdictional claims in published maps and institutional affiliations.

Received: 10 January 2018 Accepted: 26 May 2018

Published online: 07 June 2018

\section{References}

1. Ledesma-Amaro R, Lazar Z, Rakicka M, Guo Z, Fouchard F, Coq A-MC-L, et al. Metabolic engineering of Yarrowia lipolytica to produce chemicals and fuels from xylose. Metab Eng. 2016;38:115-24. https://doi. org/10.1016/j.ymben.2016.07.001.

2. Ledesma-Amaro R, Nicaud J-M. Metabolic engineering for expanding the substrate range of Yarrowia lipolytica. Trends Biotechnol. 2016;34:798-809. https://doi.org/10.1016/j.tibtech.2016.04.010.

3. Ledesma-Amaro R, Nicaud J-M. Yarrowia lipolytica as a biotechnological chassis to produce usual and unusual fatty acids. Prog Lipid Res. 2016;61:40-50

4. Řezanka T, Sigler K. Odd-numbered very-long-chain fatty acids from the microbial, animal and plant kingdoms. Prog Lipid Res. 2009;48:206-38. https://doi.org/10.1016/j.plipres.2009.03.003.

5. Degwert J, Jacob J, Steckel F. Use of cis-9-heptadecenoic acid for treating psoriasis and allergies. Patent no. WO1994021247A1. 1998.

6. Jenkins B, West AJ, Koulman A. A review of odd-chain fatty acid metabolism and the role of pentadecanoic acid (C15:0) and heptadecanoic acid 
(C17:0) in Health and disease. Molecules. 2015. https://doi.org/10.3390/ molecules20022425.

7. Forouhi NG, Koulman A, Sharp SJ, Imamura F, Kröger J, Schulze MB, et al. Differences in the prospective association between individual plasma phospholipid saturated fatty acids and incident type 2 diabetes: the EPICInterAct case-cohort study. Lancet Diab Endocrinol. 2014;2:810-8. https ://doi.org/10.1016/S2213-8587(14)70146-9.

8. Pedersen HK, Gudmundsdottir V, Nielsen HB, Hyotylainen T, Nielsen T, Jensen $\mathrm{BAH}$, et al. Human gut microbes impact host serum metabolome and insulin sensitivity. Nature. 2016;535:376-81. https://doi.org/10.1038/ nature18646.

9. Pfeuffer M, Jaudszus A. Pentadecanoic and heptadecanoic acids: multifaceted odd-chain fatty acids. Adv Nutr. 2016;7:730-4. https://doi. org/10.3945/an.115.011387.

10. Knothe G. Improving biodiesel fuel properties by modifying fatty ester composition. Energy Environ Sci. 2009;7:759-66. https://doi.org/10.1039/ B903941D.

11. Avis TJ, Boulanger RR, Bélanger RR. Synthesis and biological characterization of (Z)-9-heptadecenoic and (Z)-6-methyl-9-heptadecenoic acids: fatty acids with antibiotic activity produced by Pseudozyma flocculosa. J Chem Ecol. 2000;26:987-1000. https://doi.org/10.1023/A:1005464326573.

12. Clausen CA, Coleman RD, Yang VW. Fatty acid-based formulations for wood protection against mold and sapstain. For Prod J. 2010;60:301-4. https://doi.org/10.13073/0015-7473-60.3.301.

13. Köckritz A, Blumenstein M, Martin A. Catalytic cleavage of methyl oleate or oleic acid. Eur J Lipid Sci Technol. 2010;112:58-63. https://doi. org/10.1002/ejlt.200900103.

14. Fitton A, Goa KL. Azelaic acid. Drugs. 1991;41:780-98. https://doi. org/10.2165/00003495-199141050-00007.

15. Ingram LO, Chevalier LS, Gabba EJ, Ley KD, Winters K. Propionate-induced synthesis of odd-chain-length fatty acids by Escherichia coli. J Bacteriol. 1977;131:1023-5.

16. Wu H, San K-Y. Engineering Escherichia coli for odd straight medium chain free fatty acid production. Appl Microbiol Biotechnol. 2014;98:8145-54. https://doi.org/10.1007/s00253-014-5882-5.

17. Wu H, San K-Y. Efficient odd straight medium chain free fatty acid production by metabolically engineered Escherichia coli. Biotechnol Bioeng. 2014:111:2209-19. https://doi.org/10.1002/bit.25296.

18. Kolouchová I, Schreiberová O, Sigler K, Masák J, Řezanka T. Biotransformation of volatile fatty acids by oleaginous and non-oleaginous yeast species. FEMS Yeast Res. 2015. https://doi.org/10.1093/femsyr/fov076.

19. Zheng Y, Chi Z, Ahring BK, Chen S. Oleaginous yeast Cryptococcus curvatus for biofuel production: ammonia's effect. Biomass Bioenerg. 2012;37:114-21. https://doi.org/10.1016/j.biombioe.2011.12.022.

20. Chang HN, Kim N-J, Kang J, Jeong CM. Biomass-derived volatile fatty acid platform for fuels and chemicals. Biotechnol Bioprocess Eng. 2010;15:110. https://doi.org/10.1007/s12257-009-3070-8.

21. Fontanille P, Kumar V, Christophe G, Nouaille R, Larroche C. Bioconversion of volatile fatty acids into lipids by the oleaginous yeast Yarrowia lipolytica. Bioresour Technol. 2012;114:443-9. https://doi.org/10.1016/j. biortech.2012.02.091.

22. Fei Q, Chang HN, Shang L, Kim N, Kang J. The effect of volatile fatty acids as a sole carbon source on lipid accumulation by Cryptococcus albidus for biodiesel production. Bioresour Technol. 2011;102:2695-701. https://doi. org/10.1016/j.biortech.2010.10.141.

23. Lian J, Zhao H. Reversal of the $\beta$-oxidation cycle in Saccharomyces cerevisiae for production of fuels and chemicals. ACS Synth Biol. 2015;4:332-41. https://doi.org/10.1021/sb500243c.

24. Papanikolaou S, Galiotou-Panayotou M, Fakas S, Komaitis M, Aggelis G. Citric acid production by Yarrowia lipolytica cultivated on olive-mill wastewater-based media. Bioresour Technol. 2008;99:2419-28. https:// doi.org/10.1016/j.biortech.2007.05.005.

25. Tai M, Stephanopoulos G. Engineering the push and pull of lipid biosynthesis in oleaginous yeast Yarrowia lipolytica for biofuel production. Metab Eng. 2013;15:1-9. https://doi.org/10.1016/j.ymben.2012.08.007.

26. Qiao K, Imam Abidi SH, Liu H, Zhang H, Chakraborty S, Watson N. Engineering lipid overproduction in the oleaginous yeast Yarrowia lipolytica. Metab Eng. 2015;29:56-65. https://doi.org/10.1016/j.ymben.2015.02.005.

27. Beopoulos A, Mrozova Z, Thevenieau F, Dall MT, Hapala I, Papanikolaou S. Control of lipid accumulation in the yeast Yarrowia lipolytica. Appl Environ Microbiol. 2008;74:7779-89. https://doi.org/10.1128/AEM.01412-08.
28. Gao R, Li Z, Zhou X, Cheng S, Zheng L. Oleaginous yeast Yarrowia lipolytica culture with synthetic and food waste-derived volatile fatty acids for lipid production. Biotechnol Biofuels. 2017;10:247. https://doi. org/10.1186/s13068-017-0942-6.

29. Rodrigues $\mathrm{G}$, Pais $\mathrm{C}$. The influence of acetic and other weak carboxylic acids on growth and cellular death of the yeast Yarrowia lipolytica. Food Technol Biotechnol. 2000;12:579-1006.

30. Barth G, Gaillardin C. Yarrowia lipolytica. In: Wolf K, editor. Non conventional yeasts in biotechnology. Berlin: Springer; 1996

31. Dulermo R, Gamboa-Meléndez H, Dulermo T, Thevenieau F, Nicaud J-M. The fatty acid transport protein Fat1 $p$ is involved in the export of fatty acids from lipid bodies in Yarrowia lipolytica. FEMS Yeast Res. 2014;14:88396. https://doi.org/10.1111/1567-1364.12177.

32. Papanikolaou S, Beopoulos A, Koletti A, Thevenieau F, Koutinas AA, Nicaud J-M. Importance of the methyl-citrate cycle on glycerol metabolism in the yeast Yarrowia lipolytica. J Biotechnol. 2013;168:303-14. https ://doi.org/10.1016/j.jbiotec.2013.10.025.

33. Back A, Rossignol T, Krier F, Nicaud J-M, Dhulster P. High-throughput fermentation screening for the yeast Yarrowia lipolytica with real-time monitoring of biomass and lipid production. Microb Cell Fact. 2016;15:147. https://doi.org/10.1186/s12934-016-0546-z.

34. Gajdoš P, Ledesma-Amaro R, Nicaud J-M, Čertik M, Rossignol T. Overexpression of diacylglycerol acyltransferase in Yarrowia lipolytica affects lipid body size, number and distribution. FEMS Yeast Res. 2016. https://doi. org/10.1093/femsyr/fow062.

35. Liu L, Pan A, Spofford C, Zhou N, Alper HS. An evolutionary metabolic engineering approach for enhancing lipogenesis in Yarrowia lipolytica. Metab Eng. 2015;29:36-45. https://doi.org/10.1016/j.ymben.2015.02.003.

36. Dulermo T, Nicaud J-M. Involvement of the G3P shuttle and $\beta$-oxidation pathway in the control of TAG synthesis and lipid accumulation in Yarrowia lipolytica. Metab Eng. 2011;13:482-91. https://doi.org/10.1016/j. ymben.2011.05.002

37. Dulermo T, Tréton B, Beopoulos A, Kabran Gnankon AP, Haddouche R, Nicaud J-M. Characterization of the two intracellular lipases of Y. lipolytica encoded by TGL3 and TGL4 genes: new insights into the role of intracellular lipases and lipid body organisation. Biochimica et Biophysica Acta. 2013;1831:1486-95. https://doi.org/10.1016/j.bbalip.2013.07.001.

38. Beopoulos A, Haddouche R, Kabran P, Dulermo T, Chardot T, Nicaud J-M. Identification and characterization of DGA2, an acyltransferase of the DGAT1 acyl-CoA: diacylglycerol acyltransferase family in the oleaginous yeast Yarrowia lipolytica. New insights into the storage lipid metabolism of oleaginous yeasts. Appl Microbiol Biotechnol. 2012;93:1523-37. https ://doi.org/10.1007/s00253-011-3506-x.

39. Lazar Z, Dulermo T, Neuvéglise C, Crutz-Le Coq A-M, Nicaud J-M. Hexokinase-a limiting factor in lipid production from fructose in Yarrowia lipolytica. Metab Eng. 2014;26:89-99. https://doi.org/10.1016/j.ymben 2014.09.008.

40. Palmqvist $E$, Hahn-Hägerdal B. Fermentation of lignocellulosic hydroIysates. I: inhibition and detoxification. Bioresour Technol. 2000;74:17-24. https://doi.org/10.1016/S0960-8524(99)00160-1.

41. Chang Y-H, Chang K-S, Huang C-W, Hsu C-L, Jang H-D. Comparison of batch and fed-batch fermentations using corncob hydrolysate for bioethanol production. Fuel. 2012;97:166-73. https://doi.org/10.1016/j. fuel.2012.02.006.

42. Sagnak R, Cochot S, Molina-Jouve C, Nicaud J-M, Guillouet SE. Modulation of the glycerol phosphate availability led to concomitant reduction in the citric acid excretion and increase in lipid content and yield in Yarrowia lipolytica. J Biotechnol. 2018;265:40-5. https://doi.org/10.1016/j. jbiotec.2017.11.001.

43. Larroude M, Celinska E, Back A, Thomas S, Nicaud J-M, Ledesma-Amaro R. A synthetic biology approach to transform Yarrowia lipolytica into a competitive biotechnological producer of $\beta$-carotene. Biotechnol Bioeng. 2018;115:464-72. https://doi.org/10.1002/bit.26473.

44. Papanikolaou S, Aggelis G. Lipids of oleaginous yeasts. Part II: technology and potential applications. Eur J Lipid Sci Technol. 2011;113:1052-73. https://doi.org/10.1002/ejlt.201100015.

45. Ledesma-Amaro R, Dulermo R, Niehus X, Nicaud J-M. Combining metabolic engineering and process optimization to improve production and secretion of fatty acids. Metab Eng. 2016;38:38-46. https://doi. org/10.1016/j.ymben.2016.06.004 
46. Rigouin C, Gueroult M, Croux C, Dubois G, Borsenberger V, Barbe S, et al. Production of medium chain fatty acids by Yarrowia lipolytica: combining molecular design and TALEN to engineer the fatty acid synthase. ACS Synth Biol. 2017;6:1870-9. https://doi.org/10.1021/acssynbio.7b00034

47. Beopoulos A, Verbeke J, Bordes F, Guicherd M, Bressy M, Marty A, et al. Metabolic engineering for ricinoleic acid production in the oleaginous yeast Yarrowia lipolytica. Appl Microbiol Biotechnol. 2014;98:251-62. https ://doi.org/10.1007/s00253-013-5295-x.

48. Smit MS, Mokgoro MM, Setati E, Nicaud J-M. a,w-Dicarboxylic acid accumulation by acyl-CoA oxidase deficient mutants of Yarrowia lipolytica. Biotech Lett. 2005;27:859-64. https://doi.org/10.1007/s10529-005-6719-1.

49. Sambrook J, Fritsch EF, Maniatis T. Molecular cloning: a laboratory manual. Cold Spring Harbor: Cold Spring Harbor Laboratory Press; 1989.

50. Mlíčková K, Roux E, Athenstaedt K, d'Andrea S, Daum G, Chardot T, et al. Lipid accumulation, lipid body formation, and acyl coenzyme A oxidases of the yeast Yarrowia lipolytica. Appl Environ Microbiol. 2004;70:3918-24. https://doi.org/10.1128/AEM.70.7.3918-3924.2004.
51. Le Dall M-T, Nicaud J-M, Gaillardin C. Multiple-copy integration in the yeast Yarrowia lipolytica. Curr Genet. 1994;26:38-44. https://doi. org/10.1007/BF00326302.

52. Querol A, Barrio E, Huerta T, Ramón D. Molecular monitoring of wine fermentations conducted by active dry yeast strains. Appl Environ Microbiol. 1992;58:2948-53.

53. Fickers P, Le Dall MT, Gaillardin C, Thonart P, Nicaud JM. New disruption cassettes for rapid gene disruption and marker rescue in the yeast Yarrowia lipolytica. J Microbiol Methods. 2003;55:727-37. https://doi. org/10.1016/j.mimet.2003.07.003.

54. Browse J, McCourt PJ, Somerville CR. Fatty acid composition of leaf lipids determined after combined digestion and fatty acid methyl ester formation from fresh tissue. Anal Biochem. 1986;152:141-5. https://doi. org/10.1016/0003-2697(86)90132-6.
Ready to submit your research? Choose BMC and benefit from:

- fast, convenient online submission

- thorough peer review by experienced researchers in your field

- rapid publication on acceptance

- support for research data, including large and complex data types

- gold Open Access which fosters wider collaboration and increased citations

- maximum visibility for your research: over $100 \mathrm{M}$ website views per year

At BMC, research is always in progress.

Learn more biomedcentral.com/submissions 